\title{
Subperiosteal Osteoid Osteoma: A Rare Entity
}

\author{
${ }^{1}$ Smita Vishwanath Rathod, ${ }^{2}$ Shilpa Patel, ${ }^{3}$ Jigna Pathak, ${ }^{4}$ Niharika Swain
}

\begin{abstract}
Osteoid osteoma $(\mathrm{OO})$ is a relatively frequent benign tumor of the bone which has seldom been described in the jaws. It was originally described by Bergstand in 1930 and recognized as a distinct clinical entity by Jaffe in 1935. It is composed of osteoid and woven bone, and surrounded by a halo of reactive sclerotic bone, with an average size of the nidus less than $1.5 \mathrm{~cm}$. The lesion occurs predominantly in children, adolescents and young adults. It accounts for $3 \%$ of all primary bone tumors, and about $10 \%$ of benign bone tumors. About $80 \%$ cases of $O O$ occur in long bones while less than $1 \%$ occurs in head and neck region. The most common site is in the skull. When affecting the facial bones, they are frequently found in the mandible, the most common location being the posterior lingual surface and the mandibular angle area.

Herein, we report a rare case of $\mathrm{OO}$ of the mandible in a 16-year-old male patient with a brief literature review.
\end{abstract}

Keywords: Mandible, Nidus, Osteoid osteoma, Subperiosteal osteoid osteoma.

How to cite this article: Rathod SV, Patel S, Pathak J, Swain N. Subperiosteal Osteoid Osteoma: A Rare Entity. J Contemp Dent 2015;5(2):118-121.

\section{Source of support: Nil}

Conflict of interest: None

\section{INTRODUCTION}

Osteoid osteoma $(\mathrm{OO})$ is a distinct benign entity. The etiology of this lesion is unknown. Jaffe and Lichtenstein have suggested that the lesion is a true neoplasm of osteo-blastic derivation, ${ }^{1}$ but other workers have reported that the lesion occurs as a result of trauma or inflammation. ${ }^{6}$ Jaffe refers to the $\mathrm{OO}$ as 'Sui Genris', denoting the lesion is small with self-limiting nature. ${ }^{4}$ The lesion is typically less than $1.5 \mathrm{~cm}$ in diameter with distinct margins surrounded by a sclerotic zone of reactive new bone. The pain produced by a very small lesion is the most pathognomic and distingushing clinical feature of OO. This pain is initially dull, that may worsen at night and is relieved by nonsteroidal anti-inflammatory

\footnotetext{
${ }^{1}$ Postgraduate Student, ${ }^{2}$ Professor and Head

${ }^{3}$ Professor, ${ }^{4}$ Lecturer

${ }^{1-4}$ Department of Oral Pathology and Microbiology, MGM Dental College and Hospital, Navi Mumbai, Maharashtra, India
}

Corresponding Author: Smita Vishwanath Rathod, Postgraduate Student, Department of Oral Pathology and Microbiology, MGM Dental College and Hospital, Navi Mumbai, Maharashtra, India Phone: 02227436604, e-mail: smitarathod2012@gmail.com drugs (NSAIDS), mainly aspirin. ${ }^{7}$ Occurrence of $\mathrm{OO}$ in head and neck region is very rare, Green et al reviewed the literature and reported the total number of cases of OO of the jaws to be seven, of these four have occurred in the mandible (three were in the body and one in the condyle) and three in the maxilla (one in the antrum). ${ }^{6}$

Here, we report an unusual case of $\mathrm{OO}$ in the mandible of a 16-year-old male patient with a brief literature review.

\section{CASE REPORT}

A 16-year-old male patient reported to department of oral pathology and microbiology, MGM Dental College and Hospital, with the chief complaint of pain and swelling in lower left back region of the oral cavity since last 6 months. Patient was apparently all right 6 months back after which he started experiencing pain in lower left back region. He noticed a small nodular swelling in the same region, which had gradually increased to the present size. Pain associated with the swelling was sharp, continuous and was relieved on taking analgesics and recurred after sometimes. There was no relevant medical and family history. On general physical examination, the patient was moderately built and nourished and all the vital signs were in the normal limits. No extraoral abnormality was detected. Intraoral examination revealed a round to oval solitary swelling on lingual cortical plate in relation to 34 and 35 region with obliteration of the lingual vestibule (Fig. 1). Swelling was approximately $1 \mathrm{~cm}$ in diameter. Surface of the swelling was eroded. Borders of the swelling were indistinct. On palpation, it was firm to hard in consistency, tender nonpulsatile, nonfluctuant, noncompressible and showed no evidence of discharge on digital pressure. For the presenting complaint, an orthopantomogram (OPG) was taken which showed no pathological changes in the bone except divergent roots of 34 and 35 (Fig. 2). An excisional biopsy was done from the lesional tissue in region of 34 and 35, and sent for histopathological analysis.

Histopathologically, hematoxylin and eosin (H\&E) stained decalcified tissue section showed osteoid and woven bone in trabecular arrangement of irregular length, width and rimmed by osteoblasts (Fig. 3). Some of the trabecula showed mosaic or pagetoid pattern of reversal lines (Fig. 4). The connective tissue matrix 


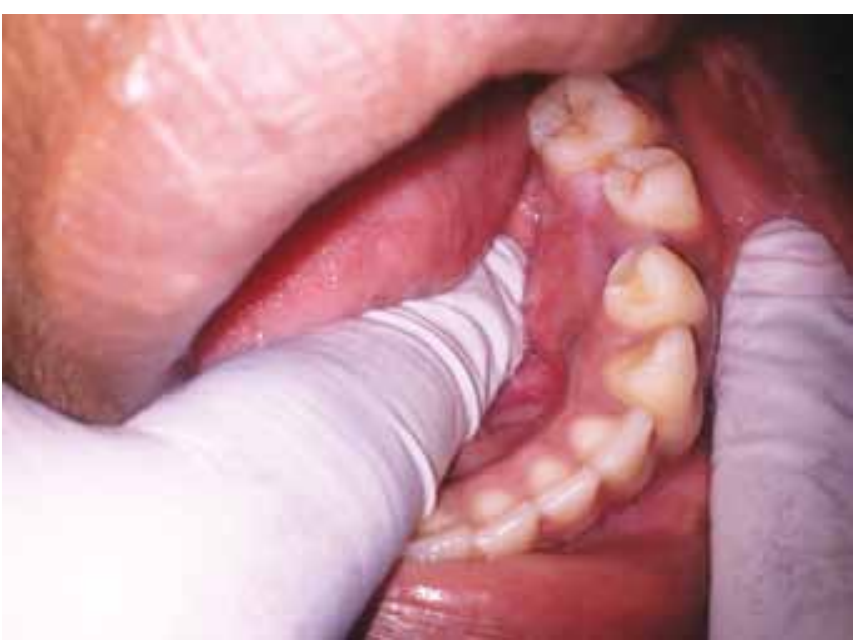

Fig. 1: Intraoral view shows a well-defined, round to oval solitary swelling on lingual cortical plate in relation to 34 and 35 obliterating the lingual vestibule. Swelling was approximately $1 \mathrm{~cm}$ in diameter

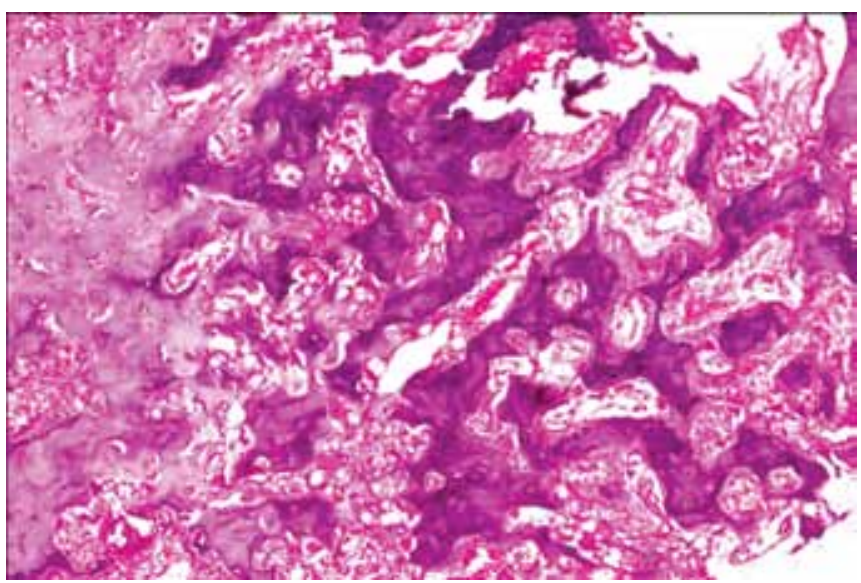

Fig. 3: Hematoxylin and eosin (H\&E) (10x) stained decalcified tissue section shows osteoid and woven bone in trabecular arrangement of irregular length and width and rimmed by osteoblasts

showed high degree of vascularity and moderate cellularity (Fig. 5). The clinical findings and the overall histopathological features were suggestive of OO.

\section{DISCUSSION}

Jaffe in 1935 described $\mathrm{OO}$ as a distinct clinical entity, and since, then 100 of cases have been published which bear his original criteria: ${ }^{9}$

- The lesion was a benign neoplasm.

- It consisted of large amounts of osteoid which became calcified.

- There was little evidence to suggest that the lesion was an inflammatory process.

- There were characteristic X-ray changes, such as focal rarefaction and reactive bone, which appeared some distance from the lesion.

- The lesion occurred most frequently in young adults.

- Pain is an outstanding feature and

- Complete removal is the treatment of choice.

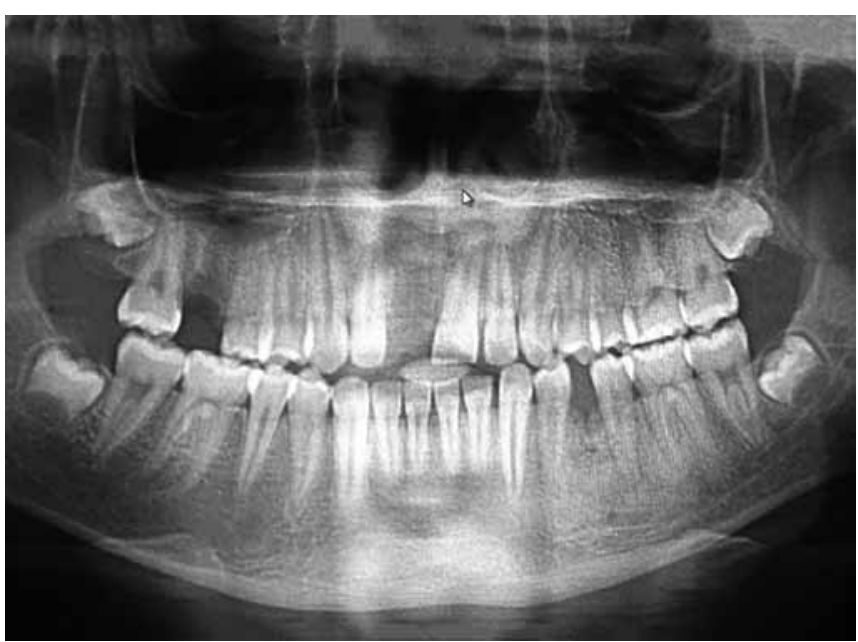

Fig. 2: An orthopantomogram showing no pathological finding except divergent roots of 34 and 35

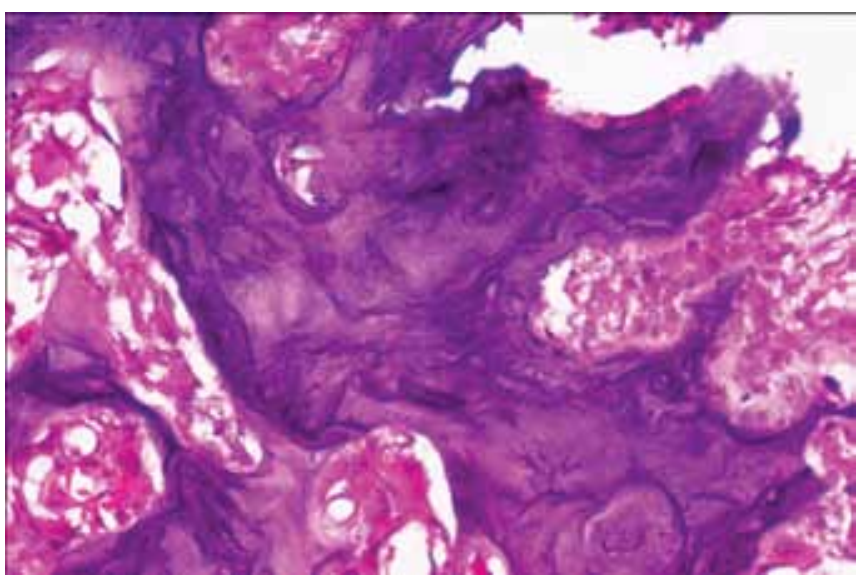

Fig. 4: In H\&E (40x) stained decalcified tissue section, some of the trabecula shows mosaic or pagetoid pattern of reversal lines

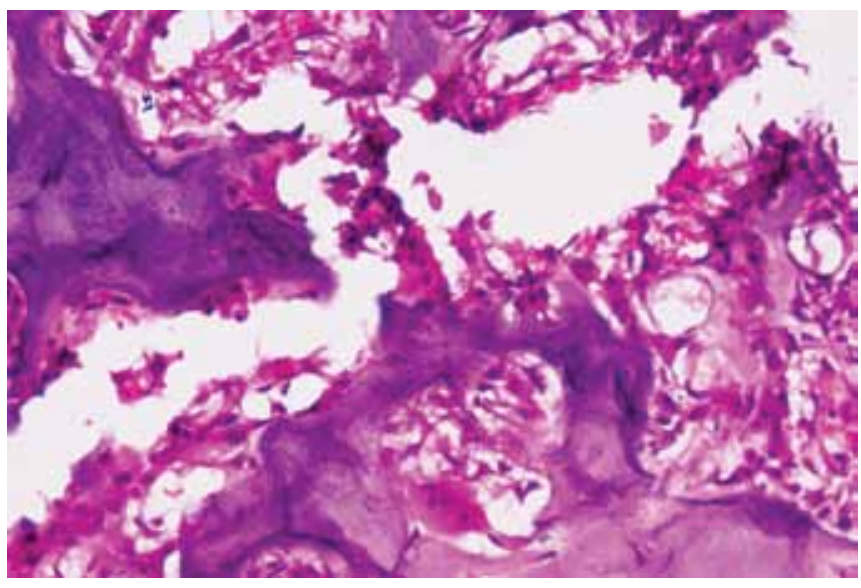

Fig. 5: Hematoxylin and eosin (H\&E) (40x) stained decalcified tissue section, the connective tissue matrix shows high degree of vascularity and moderate cellularity

The $\mathrm{OO}$ is characterized clinically as a small but painful bony lesion which has seldom been described in the jaws. Lichtenstein defined $\mathrm{OO}$ as 'a small, oval, or roundish tumor like nidus which is composed of 
osteoid and trabacula of newly formed bone deposited within a substratum of highly vascularized osteogenic connective tissue'. ${ }^{4}$ The lesion accounts for about $3 \%$ of all bone tumors, and $10 \%$ of all benign bone tumors. ${ }^{8}$ Osteoid osteoma is a benign bone tumor with $80 \%$ occurring in the long bones; majority of them in metaphysis or shaft of the femur or tibia. In the head and neck, the cervical spine is the most common site. ${ }^{7}$ When affecting the facial bones, the most common site is in the skull. Less than $1 \%$ occur in jaws. ${ }^{8}$ They are frequently found in the mandible, the most common locations being the posterior lingual surface and the mandibular angle area. ${ }^{5}$ It mainly affects adolescents and young adults. Usually, $70 \%$ of the affected young individuals are under the age of 20 , but it is very rare under the age of 5 or in adults older than 40 years. The occurrence of lesion is observed more commonly in males than females, in a ratio of approximately $2: 1$ to $3: 1.3 .^{7}$ Our case is in accordance with the literature with the lesion being seen in a 16-year-old male patient involving the left mandibular posterior lingual region in relation to 34 and 35 .

Osteoid osteoma is classified as cortical, cancellous or subperiosteal OO. Cortical lesions are the most common. The radiolucent nidus is within the cortical bone, where it is surrounded by a cortical sclerosis and thickening or solid or laminated periosteal new bone formation. Cancellous $\mathrm{OO}$ also referred to as medullary $\mathrm{OO}$ is intermediate in frequency. In this type osteosclerosis is usually mild to moderate and may be distant from the lesion. Subperiosteal $\mathrm{OO}$ is a rare form of the disease that usually presents as a rounded soft-tissue mass adjacent to a bony cortex, which it excavates. Surrounding reactive changes are usually absent in this type. ${ }^{3,4,7}$ Some authors found that subperiosteal OO like any tumors may erode into the cortex beneath. In our patient, the lesion presented as a subperiosteal $\mathrm{OO}$ in view of the fact that no pathological changes were seen radiographically and it was a soft-tissue entity.

The pathogenesis of $\mathrm{OO}$ has remained controversial. Some authors consider it as a neoplasm; others believe that it arises on an inflammatory basis; while some authors consider it as a consequence of an unusual healing process. The precise nature of the lesion remains unclear. ${ }^{10}$ The clinical hallmark of this lesion is spontaneous pain which is dull, throbbing and intermittent, but later becomes severe, constant and more pronounced during the night. These characteristics are compatible with the current case. Golding believed that the marked vascular elements were responsible for both the pain and the osseous reaction that occurs in the lesion. Jaffe regarded the curious pain seen in $\mathrm{OO}$ as being attributable to the arteriolar blood supply of the lesion. ${ }^{11}$ The evidence over the last two decades indicates that prostaglandins (especially prostaglandin E2) play a major role regarding the extremely high level of prostaglandin concentration reported in the nidus of the lesion. The dramatic pain reliefs that may occur with the administration of NSAID'S supports the theory that prostaglandins have an important pathophysiologic role for the patients with OO. ${ }^{2,3}$ In the current case, the pain was intermittent, severe in nature, and radiated to the left temporal region. Although these symptoms clinically implied a periapical inflammatory pathology, the absence of a carious tooth or any periodontal pathology ruled out the same. Pain is also a feature of malignancy, but because the lesion had a growth period of 6 months and there were no signs of paresthesia, anesthesia or perforation of the cortical bone, a benign tumor was suspected. When the physician elicits such a history, the suspicion of an osteoid OO must be strongly considered even if initial radiographs of the site fail to reveal an abnormality.

The classical radiographic appearance of $\mathrm{OO}$ is a small, radiolucent nidus, less than $2 \mathrm{~cm}$ in diameter, surrounded by a large dense sclerotic zone of cortical thickening. Evidence of a laminated periosteal reaction is often present. Jaffe emphasized that the roentgenographic features of $\mathrm{OO}$ were most important in the definitive diagnosis of the lesion. He stated that the nidus was more radiolucent than radiopaque and that it was surrounded by a reactive radiopacity that extended a variable distance from the nidus. Prichard and Mckay stated that a central opaque body which varied in density as the calcification progressed was seen. Foss et al also reported that there may be calcification or ossification of a portion or all of the radiolucent nidus. Computed tomography has been recommended to be one of the most useful modalities to diagnose bone tumors in the complex anatomical site of the jaws. The characteristic features of $\mathrm{OO}$, such as a radiolucency with central calcification, located in the cortical bone with surrounding sclerotic bone are best elucidated by CT. Bone scanning is considered a useful adjunctive test in some cases because the rich bone-producing $\mathrm{OO}$ is associated with a 'hot' radionuclide bone scan. Radionuclide scanning is a sensitive technique, and findings may be positive before radiographic changes are apparent. ${ }^{4,12}$ However, since in our case no radiologic abnormality was evident, we suspected the lesion to be a subperiosteal $O O$ which is an extremely rare entity.

The histopathological appearance of $\mathrm{OO}$ varies with the age of lesion and its site. Nidus, which contains osteoid tissue, varying in degree of calcification, and/or bone trabeculation with large vascular systems, is characteristic feature of OO. Formation of definite trabeculae occurs, particularly in older lesion, outlined by active osteoblasts. Large sheets or irregular trabeculae reveal mineralized material that demonstrates prominent reversal lines. Foci 
of bone resorption are also usually evident. The overlying periosteum exhibits new bone formaton, and in this interstitial tissue collections of lymphocytes may be noted. Multinucleated giant calls are rarely seen. Our microscopic findings were in agreement with the literature. ${ }^{6}$

In the differential diagnosis, we considered fibroosseous lesions and tumors of bony origin. One of the challenging issues with $\mathrm{OO}$ is differentiating the tumor from osteoblastoma (OB), because both are closely related entities. There are no specific histological criteria to distinguish $\mathrm{OO}$ from OB. Some authors believe that, in spite of the similarity in histopathologic features, they are distinguishable by clinical symptoms, size, site and radiological appearance. Ossifying fibroma has some similar clinical and radiographic features to $\mathrm{OO}$, it is usually painless and lacks the nidus. Fibrous dysplasia is a poorly defined lesion showing characteristic chinese letter pattern histopathologically and being continuous with the host bone; neither of which were seen in this case. Paget's disease shows pathognomonic histologic feature of a mosaic pattern of bone formation but is mostly seen in older patients unlike in our case which showed the mosaic pattern in a young adult.

There are three main approaches for the treatment of OO, namely: conservative treatment, surgical excision and percutaneous excision. Osteoid osteoma is first treated conservative methods (NSAID) followed by surgical excision or curettage, but newly developed minimally invasive techniques, such as CT-guided percutaneous radiofrequency thermal ablation and laser photocoagulation, have become the methods of choice for the treatment. There is general agreement in the literature that the complete excision of an $\mathrm{OO}$ is the treatment of choice. Incomplete excision of the lesion leads to the recurrence of symptoms. Curettage of the cavity is not advised, because if it is not thoroughly performed the lesion is liable to recur. The tumor has no malignant potential. There is fairly good circumstantial evidence that spontaneous regression may occur in at least some untreated cases. ${ }^{6}$ Our case was treated with surgical excision with no recurrence reported in a 3 years follow-up period.

\section{CONCLUSION}

Osteoid osteoma is a benign bone lesion that occurs very rarely in the jaw bones. Herein, we reported a case of subperiosteal $\mathrm{OO}$ that occurred in the mandible of a young adult. The lack of knowledge concerning the genesis of $\mathrm{OO}$ and its confusion with similar looking lesions in the bone make accurate compilation of data concerning the lesion a difficult task. It is mandatory to correlate the proper clinical, radiographic and histopathological examination to arrive at the correct diagnosis. It is obvious that the small number of reported cases of $\mathrm{OO}$ in jaw bone hinders any lasting conclusions concerning this lesion's behavior in the jaws. Thus, it necessitates an increased awareness among the dentists worldwide to report additional cases in literature.

\section{REFERENCES}

1. Golding JSR. The natural history of osteoid osteoma: with a report of twenty cases. J Bone Joint Surg 1954;36(2):218-229.

2. Rahsepar B, Nikgoo A, Fatemitabar SA. Osteoid osteoma of subcondylar region: case report and review of the literature. J Oral Maxillofac Surg 2009;67(4):888-893.

3. Kitsoulis P, Mantellos G, Vlychou M. Osteoid osteoma. Acta Orthop Belg 2006;72(2):119-125.

4. Walia C, Devi P, Thimmarasa VB, Jayadev S. Osteoid osteoma of the mandible: a rare entity. J Indian Acad Oral Med Radiol 2010 Jul-Sep;22(3):162-164.

5. Chaudhary M, Kulkarni M. Osteoid osteoma of mandible. J Oral Maxillofac Pathol 2007;11(2):52-55.

6. Shafer WG, Hine MK, Levy BM. A Textbook of Oral Pathology. 7th ed. Elsevier. p. 155-156.

7. Rashmishree K, Kruthika R, Venkatesh SG, Naikmasur G, Burde K. Osteoid osteoma of the mandible. E Journal of Dentistry 2014;4(1):565-570.

8. Ida M, Kurabayashi T, Takahashi Y, Takagi M, Sasaki T. Osteoid osteoma in the mandible. Dentomaxillofac Radiol 2002;31(3):385-387.

9. Jaffe HL. Osteoid osteoma. Arch Surg 1935;31(5):709-728.

10. Lee EH, Shafi M, Hui JH. Osteoid osteoma, a current review. J Pediatr Orthop 2006;26(5):965.

11. Singh A, Solomon MC. Osteoid osteoma of the mandible: a case report with review of the literature. J Dental Sciences (2012). Available at: http://dx.doi.org/10.1016/j.jds.2012.10.002.

12. Kayser, et al. Evidence of the subperiosteal origin of osteoid osteomas in tubular bones: analysis by CT and MR imaging. AJR: 170, March 1998. 